\title{
La Psicología en la escritura: una visión general
}

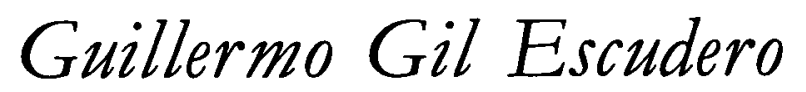

Universidad Complutense de Madrid

La durabilidad de la escritura permite la permanencia de las ideas, así como su transmisión intergeneracional e interhumana. Si la transmisión de los conocimientos a través del tiempo se realizase solamente mediante el lenguaje oral, se perdería gran parte de la información $\mathrm{y}$, asimismo, gran parte de la restante se vería modificada por cada una de las sucesivas versiones. De modo que la escritura ha sido el instrumento que la especie humana ha utilizado para crear una memoria permanente común del conocimiento, la ciencia, y de su propio desarrollo e historia, lo que ha originado que se considere en ocasiones como el mayor invento humano. Por otro lado, el desarrollo de la informática, al permitir el manejo de modo sencillo de grandes cantidades de información y la impresión automática de textos, junto con el desarrollo de la inteligencia artificial, facilitará en el futuro las tareas más arduas del proceso de escritura.

Dada la importancia de la escritura para la adquisición de información, la culturización y el aprendizaje en general, resulta imprescindible su dominio de cara a alcanzar los niveles de desarrollo personal y social propios del hombre de las sociedades desarrolladas. Asimismo, su dominio es de suma importancia en el ámbito educativo, ya que habitualmente el progreso académico se demuestra por medio del lenguaje escrito, que resulta de este modo ser, a la vez, un objeto de estudio y una parte relevante del proceso educativo.

Desde esta perspectiva, la escritura permite reconsiderar y repensar lo que el escritor intenta decir, contribuyendo de ese modo a la organización y claridad del pensamiento. De modo que la escritura constituye un proceso de descubrimiento, de exploración y de creación de ideas a través del cual éstas se extienden y refinan, al ser éste un proceso de descubrimiento que implica mantener y 
seleccionar en la mente las ideas en una secuencia lógica, convirtiéndose así la escritura en un instrumento para construir la realidad y actuar frente al mundo.

El término «escritura» se emplea de forma ambigua, tanto para denominar la actividad proceso de escritura como para designar el producto de dicha actividad. Esta ambigüedad se ve reflejada, por ejemplo, en el hecho de que mientras que unas veces se utiliza este término para referirse a los aspectos caligráficos de la escritura, otras se utiliza para referirse a temas tan distintos como la escritura creativa (Sovik, 1975).

El concepto de escritura, asimismo, no es un concepto bien delimitado, ya que en algunas ocasiones se consideran como escritura solamente los aspectos motores de la tarea, mientras que en otros se consideran como tal los procesos de pensamiento. Habitualmente se intenta delimitar el concepto de escritura a partir de dos perspectivas. La primera considera la escritura como un producto. Por ejemplo, según Cohen (1958), «la escritura consiste en una representación visual duradera del lenguaje, que lo convierte en algo que se puede conservar y trasladar de un sitio a otro. La condición esencial de la escritura es que se puede transmitir». La segunda conceptualiza la escritura como un proceso. Britton, Burguess, Martin, McLeod y Rosen (1975) consideran que se trata de un acto premeditado y apoyado por la imaginación, no existiendo un oyente directo $y$, por tanto, tampoco retroalimentación contemporánea como en el habla. Para Gelb (I952), la escritura implica un rango de procesos psicológicos influidos por la experiencia del escritor, variable que afecta el grado en que operan los procesos integrativos. Este acto privado se diferencia de otras actividades cognitivas sofisticadas en el grado en que el contexto ambiental gobierna el procesamiento mental (Bracewell, 1980 ).

Muchos autores defienden que la escritura es un sistema de símbolos, es decir, un sistema de símbolos del lenguaje oral, que, a su vez, es un sistema de símbolos (Stallard, 1977; Ajuriaguerra y Auzias, 1980; Vygotsky, 1983). Para Vygotsky (1983), con el tiempo, este simbolismo de segundo orden se convierte en un simbolismo de primer orden, o en un simbolismo directo. Pero si la escritura fuera una mera representación visual del lenguaje hablado, entonces aprender a leer no implicaria aprender un nuevo sistema de comunicación, sino solamente aprender a transferir una habilidad lingüistica ya adquirida de un medio (habla) a otro (escritura): de sonidos a símbolos visuales (Stubbs, 1980). De modo que se considera que, una vez que el lenguaje escrito existe, se hace parcialmente autónomo, como se demuestra con la diferencia entre la pronunciación de una palabra como tal y la pronunciación de su deletreo. Por otro lado, la relación entre la escritura y el lenguaje oral ha cambiado en función de los propios cambios que se han producido en los usos y formas del lenguaje escrito (Stubbs, 1983). 


\section{ESTUDIO HISTORICO Y ONTOGENETICO DE LA ESCRITURA}

La evolución de la escritura se ha estudiado desde dos puntos de vista: en cuanto a su desarrollo en la humanidad, o estudio histórico, y en relación a su desarrollo individual, o estudio ontogenético.

El estudio histórico de la escritura, llevado a cabo por disciplinas como la filología, la antropología, la arqueología y la historia, muestra que la escritura es un medio de comunicación peculiar del hombre que aparece relativamente tarde en la historia del desarrollo humano y que ha sido creado por la necesidad cultural de registrar las expresiones habladas y darles permanencia a través del espacio y del tiempo (Russell, i982).

Generalmente se han distinguido tres clases de sistemas de escritura, que, ordenados según el desarrollo histórico, son el sistema pictográfico, el ideográfico y el fonográfico, aunque existen otras clasificaciones (Gelb, I95 2; Haas, 1976; Henderson, 1982).

En la escritura pictográfica el símbolo está más o menos directamente relacionado con la apariencia visual de su referente, teniendo un valor semántico, por lo que intenta transmitir el mensaje de la misma forma que lo hacen los dibujos en los libros infantiles (Brown, 1958; Smith, 1973; Russell, 1982). En la escritura ideográfica el símbolo visual está directa y arbitrariamente relacionado con la «idea» del referente, utilizándose los dibujos de forma simbólica, sin elementos fonéticos en su forma pura, por lo que los ideogramas permiten una comunicación entre lenguajes y poseen la ventaja del impacto visual directo y de la inmediatez (Smith, 1973; Russell, 1982). Por tanto, ni los símbolos pictográficos ni los ideográficos tienen conexión con el lenguaje hablado.

El sistema fonológico, que para algunos, como Stubbs (i980) y Paivio y Begg (1981) es el único sistema de escritura, utiliza los símbolos para indicar la pronunciación de unidades de sonido (Smith, I 973; Gibson y Levin, 1975; Stubbs, 1980; Paivio y Begg, I981; Russell, 1982). Mientras que en el sistema logográfico o morfémico los símbolos representan palabras o morfemas, en el sistema silábico los símbolos representan la sílaba, y en la escritura alfabética están más o menos relacionados directamente con unidades de sonido individuales, de forma que se da una relación entre la fonología del habla y la ortografía de la escritura.

En cuanto al desarrollo individual, el lenguaje escrito es el último sistema simbólico que adquiere el niño y puede considerarse como el nivel más alto del comportamiento verbal del ser humano (Myklebust, 1964). Sólo se puede alcanzar la escritura una vez logrado cierto nivel de desarrollo intelectual, motor, sensorial y afectivo (Ajuriaguerra y colbs., 1973; Ajuriaguerra y Auzias, 1980), así como un determinado nivel de pensamiento abstracto, autorresponsabilidad y madurez de automotivación (Lundsteen, 1976). Es necesario que todos los niveles precedentes de habilidades lingüísticas (escucha, habla, lectura) se hallan establecido previamente (Myklebust, 1965), de modo que la existencia de un problema en 
cualquier área del lenguaje ya adquirido puede afectar el desarrollo de las destrezas de escritura (Bain, 1976; Cicci, 1980).

\section{CAMPOS DE ESTUDIO PSICOLOGICO DE LA ESCRITURA}

El estudio psicológico de la escritura puede dividirse en cuatro campos: el estudio del proceso y del producto de la escritura, el análisis de su relación con otros procesos, el estudio de la relación de la escritura con variables diferenciales y, por último, el área de su evaluación. Es importante tener en cuenta que no existen límites definidos entre estos campos, sino que los diferentes estudios tratan temas que se incluyen en varias áreas al mismo tiempo.

El campo de estudio del producto y del proceso de la escritura abarca, a su vez, un conjunto de subáreas: el análisis de los prerrequisitos o habilidades que debe poseer el niño para la adquisición y el desarrollo de la escritura (Alvarez y Orellana, 1979; Salminen, 1979; Ajuriaguerra y Auzias, 1980; Stallard y Stallard, I 980; Temple, Nathan y Burris, 1982); el estudio del desarrollo de la escritura, que se ocupa de la evolución de la misma desde el momento en que el niño comienza a escribir hasta que se considera que ha adquirido el dominio de la escritura (Myklebust, 1965; Britton, Burgess, Martin, McLeod y Rosen, I975; Graves, I979; Fagan, 1981; Gundlach, 1982; Temple, Nathan y Burris, 1982; Martlew, 1983; Bereiter y Scardamalia, 1983; Goès y Martlew, 1983; Wiseman, I984); el estudio de los procesos implicados en la escritura, generalmente estudiados a través de la observación de determinadas conductas, como las pausas o los informes verbales (Emig, I 971; Graves, I 973; Stallard, I 974; Perl, 1978; Beaugrande, I 982 ; Black; I982; Nystrand, 1982; Mosenthal, Tamor y Walmsley, I 983 ; Beach y Bridwell, I984; Benton, Glover y Plake, 1984); el área de los trastornos que se presentan en la adquisición y desarrollo del lenguaje escrito, incluyendo tanto aquellos propios de la escritura como los que se deben a problemas en otras áreas de conducta (Myklebust, 1973; Vachek, I973; Weigl, 1975; Auzias, 1978; Bradley, 1980; Marcie, 1983; Kelly y Whitehead, I983; Blair y Crump, 1984); el análisis de los tratamientos de dichos trastornos, que se caracteriza por su diversidad (Olivaux, I 971; Lobrot, 1972; Ajuriaguerra, 1978; Gilligham y Stillam, I977; 'Tsvêtkova, 1977; Blumenstock, 1979, y, por último, el tema de la enseñanza, tanto en el ámbito escolar como fuera de él, a menudo estudiado con el objeto de crear y examinar la eficacia de distintos programas de instrucción (Elbow, 1973; Hailey, 1978; Donovar y McClelland, 1980; Tamor, Bond y Matz, 1980; Stanford y Smith, 1981; Pringle y Freedman, 198r; Flower, 1981; Phelps-Gunn y Phelps-Teresaki, 1982; Allen, 1984; Langer, 1984; Harrop y McCann, 1984).

En cuanto a la relación de la escritura con otros procesos, ésta se ha relacionado frecuentemente con el habla, ya que en ambos se utiliza el lenguaje, pero no existe acuerdo entre los distintos autores respecto a si la escritura es o no un sistema simbólico del habla y, por tanto, secundario (Schallert, Kleinman y Rubin, 1977; Farrel, 1978; Rubin, 1978; Cameron, 1979; Cayer y Sacks, 1979; Kroll y 
Vann, I981; Tannen, 1981, 1982; Nauclér, 1983). De igual modo, se relacionan la escritura y la lectura, a veces considerándose como procesos inversos, y otras como procesos totalmente distintos, aunque mantengan una determinada relación (Smith, 1971; Frith, 1979; Read, 1981; Trosky y Wood, 1982). Por último, también se ha estudiado la relación entre el primer y el segundo lenguaje (Edelsky, 1982; Kayfetz, 1982).

El tercer grupo de estudios abarca los que relacionan la escritura y su desarrollo con variables diferenciales, tales como sexo, clase social, etc... (Poole, 1972; Owens, 1973; Hausner, 1975; Kramer, 1977; Caroselli, 1980; Poole, 1983; Freedman, 1983).

Finalmente, el campo de estudio de la evaluación de la escritura está muy estrechamente relacionado con todos los anteriores, ya que a todos ellos les resulta necesario o bien medir aspectos aislados de la misma, o bien evaluar sus características o calidad de modo global.

\section{EL MARCO DEL ESTUDIO COGNITIVO DE LA ESCRITURA}

A pesar de la necesidad de establecer un marco que defina cuál es o debe ser el campo de investigación psicológica de la escritura, hasta el momento sólo ha habido un intento de definir el mismo (Frederiksen y Dominic, 198 I b). En su propuesta, Frederiksen y Dominic se proponen integrar lo que para ellos son las cuatro perspectivas más relevantes del estudio de la escritura y que se caracterizan por considerar fundamentalmente la escritura, bien como una actividad cognitiva, como una actividad lingüística, como una actividad comunicativa, o bien como una actividad contextual.

Otro marco posible para la organización del estudio de la escritura es el que se presenta en la figura I y que intenta integrar los componentes principales que se tienen en cuenta, tanto en la teoría como en la investigación actual, desde una perspectiva eminentemente cognitiva, ya que los enfoques más recientes sobre la escritura utilizan habitalmente los constructos teóricos y los métodos de investigación de la moderna psicología cognitiva.

Se contemplan en este esquema cuatro componentes globales: los procesos cognitivos del escritor, el producto de la escritura y las variables, tanto internas como externas, que influencian y restringen dichos procesos.

Los procesos cognitivos del escritor hacen referencia a los planes y las metas que el escritor utiliza, así como a los procesos de pensamiento reales durante la escritura, que se estructuran en diferentes estrategias diseñadas para hacer frente a las demandas y requisitos de la tarea. Estos procesos se combinan en tres grandes actividades: la "preescritura», "planificación» o «ideación», a través de la cual el escritor genera, selecciona y organiza el contenido a transmitir, y planifica la propia tarea de escritura; la «escritura», "traducción", "transcripción» o "articulación», mediante la que el escritor transforma en lenguaje escrito los contenidos y su organiza- 


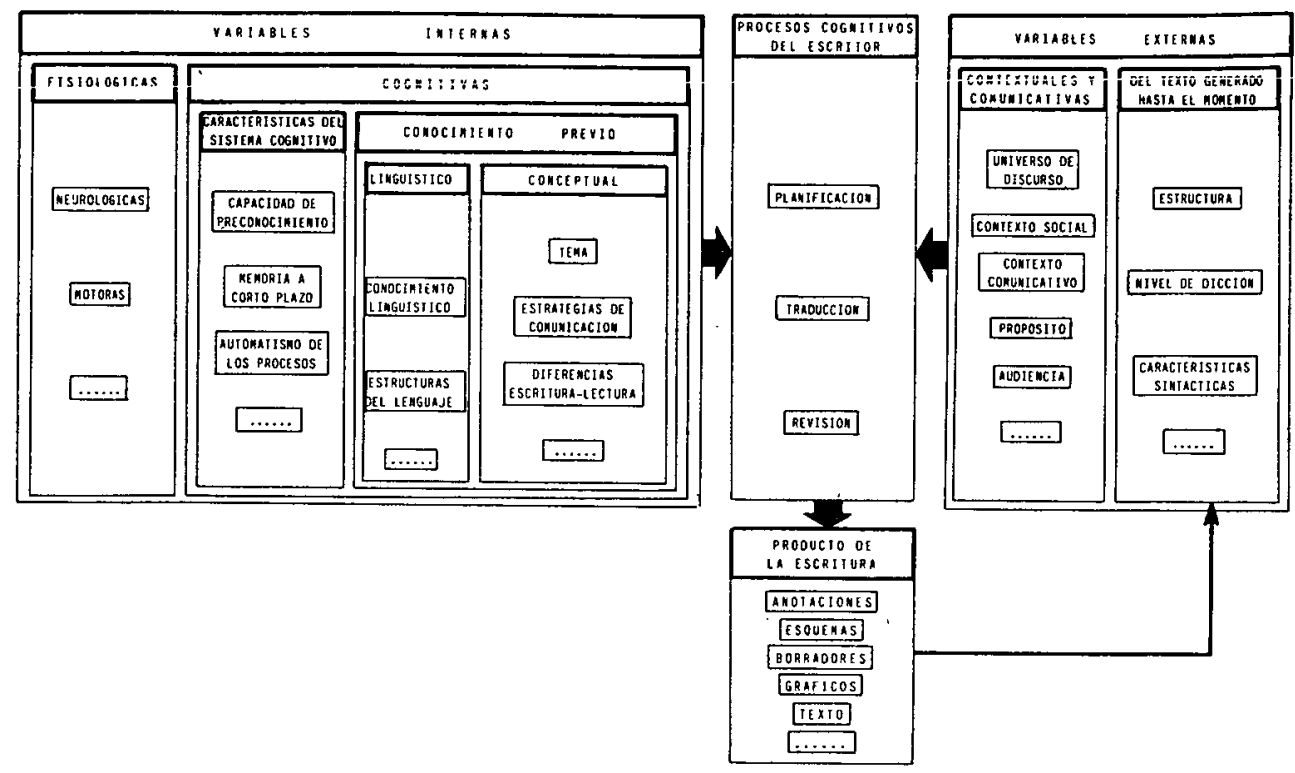

FIGURA 1

Procesos cognitivos del escritor, producto de la escritura y variables internas y externas que influencian $y$ restringen dichos procesos cognitivos. Esquema de un marco de estudio de la escritura

ción, y la "posescritura», "relectura» o «revisión», que el escritor utiliza para comprobar si el resultado escrito se adecúa a los objetivos comunicativos que pretende alcanzar.

El componente del producto escrito hace referencia tanto al texto final como a los sucesivos resultados que se generan a lo largo del proceso de escritura, tales como anotaciones, esquemas, borradores y dibujos. Es importante señalar que estos resultados, desde el momento mismo de su producción, pasan a actuar como un estímulo externo que afecta a los procesos, constituyendo el ciclo de retroalimentación en el que se basa fundamentalmente el proceso de revisión. En este marco, el análisis del producto escrito está estrechamente relacionado con las teorías del texto y del discurso (Kinneavy, 1971; Freedle, 1977, 1979; Van Dijk, 1977; Beaugrande, 1980; Flammer y Kintsch, 1982; Nystrand, 1982; Cooper, 1982).

Otro gran componente está formado por las variables internas que afectan a los procesos cognitivos del escritor. Se pueden diferenciar en éstas dos grandes bloques: las variables fisiológicas, dentro de las cuales se incluirían los aspectos neurológicos y de coordinación y de control motor, que presentan especial relevancia a la hora del estudio de los trastornos de la escritura (Rosenthal, I 977; Marcie, I 983) y las variables cognitivas. En cuanto a estas últimas, se han considerado especialmente, tanto en la teoria como en la investigación, las características del sistema cognitivo, tales como la capacidad de procesamiento, la atención, el papel de la memoria y el automatismo de los procesos, que limitan las posibili- 
dades de procesamiento y determinan la estructuración de los planes de escritura, asi como las bases de conocimiento previo que el escritor utiliza durante la tarea. Dentro de esta amplia categoría se han considerado dos tipos: el conocimiento lingüístico, especialmente el conocimiento sobre las formas, principios y estructuras del lenguaje y su uso, y sobre las convenciones ortográficas, morfológicas, léxicas, sintácticas y del discurso; y el conocimiento conceptual, tanto sobre el tema de escritura como sobre las diferencias de naturaleza entre los sistemas de comunicación hablado y escrito, como sobre las estrategias de comunicación y de planificación, traducción y revisión propias del lenguaje escrito.

El último componente lo constituyen las variables externas. Un grupo es el formado por las contextuales y comunicativas, que obligan a integrar su actividad en contextos de distintos niveles: universo de discurso, contexto social, contexto comunicativo, y que limitan las funciones y propósitos de la misma. Estas exigen al escritor tener la capacidad de situarse en el papel del lector y realizar el cambio de roles lector-escritor rápida y eficazmente. Al respecto, tanto en la teoría como en la investigación, se ha tenido en cuenta en especial el sentido de la audiencia y su adquisición (Britton, Burgess, Martin, Mcleod y Rosen, 1975; Britton, 1982). Otra importante variable externa, como se ha mencionado más arriba, está constituida por los propios resultados escritos, donde son de especial relevancia la estructura, las características sintácticas y el nivel de dicción del texto generado hasta cl momento.

Por otro lado, hay que tener en cuenta que la actividad de escritura se inscribe en el marco más amplio de las habilidades psicolingüísticas de comunicación. En la figura 2 se presenta el esquema de un marco de este tipo. Podemos considerar en este marco dos ciclos distintos de comunicación. El primer ciclo y más básico sería el ciclo de la comunicación hablada, en el que el cmisor, a partir del significado y a través del proceso de producción, genera el habla. Este resultado del habla actúa como estímulo para el receptor, que, a través del proceso de comprensión, trata de obtener como resultado el significado que se ha intentado transmitir. Este çiclo básico sería el utilizado en aquellas sociedades que carecen de escritura.

El ciclo del lenguaje escrito requiere la intervención de todos los elementos del ciclo básico, en el que se basa y del que es una extensión. En este ciclo, a partir del significado que se intenta comunicar y con la mediación de los procesos generales de producción y los especificos del habla, a través del proceso de escritura se genera como resultado el texto, que a su vez sirve de estímulo receptor para, a través de los procesos de lectura, en los que juega un papel importante el habla, y los de comprensión, recuperar el significado transmitido. Las sociedades desarrolladas se caracterizan por el uso extensivo de este segundo ciclo.

De modo que para el estudio de la actividad de escritura resulta especialmente imprescindible el análisis integrado de las relaciones entre estos procesos y los estímulos y resultados de los mismos, dado que en el proceso de escritura cada uno de ellos desempeña un 


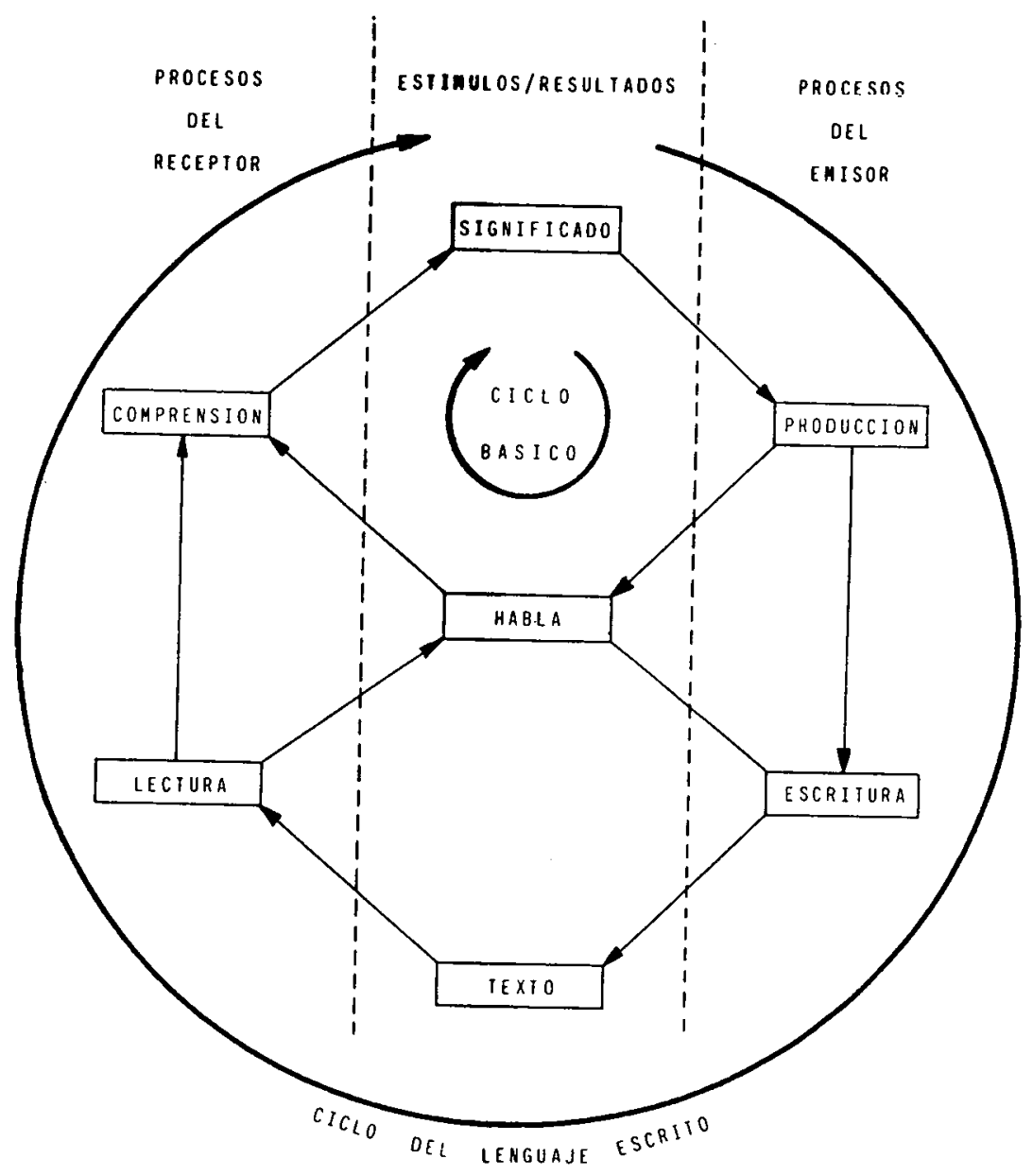

Figura 2.

Esquema de un marco de babilidades psicolingüisticas de comunicación.

papel trascendental. Desde esta perspectiva es relevante el estudio de las características del significado, ya que la propia naturaleza de la escritura y las restricciones que lleva asociadas hacen que los contenidos y organización que se transmiten a través del ciclo del lenguaje escrito sean, en muchos casos, distintos de los que se transmiten a través del ciclo del lenguaje hablado. Por razones similares, los procesos de producción y comprensión del ciclo del lenguaje escrito, aunque regidos por los mismos principios generales que los del hablado, están condicionados por la descontextualización del lenguaje escrito, obligando al escritor a tener en cuenta de modo más acentuado los mecanismos que dan coherencia al texto $y$ obligando al receptor a realizar complejas tareas de inferencia. Por otro lado, el estudio del texto es importante no solamente por ser el resultado de la tarea de escritura, sino también por el papel que ejerce como una memoria externa que interviene a lo largo del 


\section{Estudios}

proceso y cuyas propias características le condicionan. Asimismo, la lectura interviene de modo decisivo en la tarea global de escritura, al ser el proceso a través del cual se extrae la información necesaria para la actividad de revisión. Por último, en este esquema el habla ocupa un papel central debido a la importancia de su actividad mediadora, tanto para la tarea de escritura como las tareas de lectura implicadas en la misma.

En conclusión, un concepto amplio de la actividad de escritura implica su consideración como una destreza constituida por el funcionamiento integrado y eficaz del resto de las habilidades psicolongüísticas, en el que los grados y modos de participación de dichas habilidades, así como las relaciones e interacciones que se dan entre las mismas, están reguladas por los requisitos y objetivos de la tarea. La utilización integrada de estas habilidades para alcanzar los objetivos comunicativos en función de las demandas y requisitos contextuales, dan lugar a la generación de estrategias concretas para cada tarea de escritura y permiten, asimismo, la generación de un amplio rango de estrategias individuales, lo que explica las grandes diferencias individuales que se observan en la realización de esta tarea compleja.

A continuación se exponen en este dossier tres trabajos que se inscriben en el marco de estudio de la escritura. En primer lugar, se presenta una revisión de los modelos del proceso de escritura, en la que se analiza tanto el desarrollo de los mismos como los supuestos y características de los modelos cognitivos actuales. En segundo lugar, se presenta una revisión del estado actual del tema de la evaluación de la escritura, en la que se examinan el objeto de la evaluación y las clasificaciones usuales de los sistemas de evaluación de la escritura, así como las características y los problemas que plantean los mismos. Por último, se presenta un trabajo experimental dirigido a la construcción de un sistema objetivo de evaluación de la escritura, que sea útil para estudiar el desarrollo de dicha habilidad y que pueda ser utilizado con distintos grados de especificidad.

\section{Referencias}

Ajuriaguerra, J., y cols: La escritura en el niño. Vols. I y II. Barcelona, Edit. Laia, 1973.

Ajuriaguerra, J., y Auzias, M.: «Preconditions for the development in the child». En J. HARTIEY: The psychology of written communication. Selected readings. Londres, Kogan Page, 1980.

AII.EN, G. J.: «Using a personalized system of instruction to improve the writing skills of undergraduates». Teaching of Psycbology, 1984, 11 (2), $95-98$.

Ai.varez, A., y OREI.l.ANA, E.: "Desarrollo de las funciones necesarias para el aprendizaje de la lectura y la escritura de acuerdo con la teoria de Piaget: II». Revista Latinoamericana de Psicologia, 1979, 11 (2), 249 -259. Auzias, M.: Los trastornos de la escritura infantil. Problemas generales. Bases para su reeducación. Barcelona, Edit. Laia, $197^{8}$.

BAIN, A. M.: "Written expression - the last skill acquired». Bulletin of the orton Society, 1976, 26, 75-95.

BEACH, R., y Bridwell, L. S.: New directions in composition research. Londres, Guilford, 1984.

Beaugrande, R. DE: Text, Discourse and Process. Norwood, Ablex, 1980.

BEAUGRANDE, R. DE: «Linguistic and cognitive processes in development writing». International Review of Applied Linguistic, 1982 .

BEnTON, S. L.; Glover, J. A., y Plake, B. S.: «Emploing adjunct aids to facilitate elaboration in writing". Research in the Teaching of English, 1984, 18 (2), 189-200. 
BEREITER, C., y SCARDAMAIIA, M.: “Development of cognitive structures for writing». Bebavioral and Brain Sciences, $1983,6(1)$.

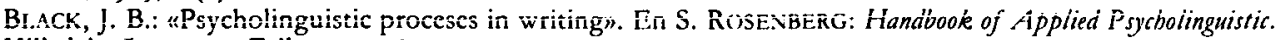
Hillsdale, Lawrence Erlbaum, 1982.

Bi.siR, T. K., y KRUMP, W. D.: «Effects of discourse mode on the syntactyc complexity of learning disabled students' written expresion", Learning Disability Quarterly, 1982, 7, 19-29.

Blumenstock, L.: Propbylaxe der Lese- und Rechtschreibschwäcbe: Erstellung und Erprobungeines spracbl-akust. Trainingsverfabrens zur Verbesserung d. grundlegenden Leseund Rechtscbreibfäbigkeit der Schüller in 1 . Scbuljabr. Weinheim, Beltz, I979.

BRACEWEL.l, R. J.: «Writing as a cognitive ability». Visible Language, 1980, XIV (4), 400-422.

BRADI.EY, L.: Reading, writing and spelling problems. Edimburgo, Churchill Livingstone, 1980.

BRITTON, J.: «Spectator role and the beginnings of writing". En M. NYSTRAND (Ed.): What writers know. The language, process and structure of written discourse. Cap. 7. Nueva York, Academic Press, 1982.

Britton, J.; BURgess, T.; MARTIN, N.; MCLeOD, S., y ROSEN, H.: The development of writing abilities $(I I-18)$. Londres, McMillan Education, 1975.

BRown, R.: Words and things: an introduction to language. The McMillan Company, 1958.

CAMERON, D. B.:The relationship of written and oral languae in cbildren age nine, ten and eleven. The University of Alberta, 1979 .

CAROSELLI, M.: The effect of parental involvement on the writing skills and attitudes of secondary students. The University of Rochester, 1980.

CAYER, R. L., y SACKS, R. K.: "Oral and written discourse of basic writers: similarities and differences". Research in the Teaching of English, 1979, 13, 121-128.

CiCCI, R.: "Written language disorders". Bulletin of the Orton Society, 1980, 30, 240-251.

COHEN, M.: La grande invention de P'écriture et son évolution. París, Imprisserie Nationale, 1958.

COOPER, M. M.: "Context as vehicle: Implicatures in writing". En M. NYSTRAND (Ed.) What writters know. The language, process and structure of written discourse. Cap. 5. Nueva York, Academic Press, 1982.

Donovin, T. R., y MCCl.EL.L.AND, B. W. (eds.): Eight approaches to teaching composition. Urbana, National Council of Teachers of English, 1980.

E DEI.SKY, C.: "Writing in a bilingual program: the relation of $L_{1}$ and $L_{2}$ texts». Tesol Quartely, 1982,16 (2).

EI.BOW: Writing without teachers. Londres, Oxford University Press, 1973.

EMIG, J.: The composing processes of twelfth graders. Urbana, National Council of Teachers of English, 1971.

FAGAN, W. T.: "A longitudinal study of grade five and grade eleven student's written language and a comparison with teacher's written language». The Alberta Journal of Educational Research, 1981, 27 (3).

FidMMER, y Kinisch, W.: Discourse Processing. Amsterdam, North-Holland, 1982.

FARREL, T. J.: «Differentiating writing from talking». College Composition and Communication, 1978, 29, 346-350.

FLOWER, L: Problem-Solving strategies for writing. Nueva York, Harcourt Brace Jovanovich, 1981.

Frederiksen, C. H. y DominiC, J. F.: «Introduction: Perspectives on the activity of writing". En C. H. FREDERIKSEN y J. F. DOMINIC (Eds.): Writing: The nature, development and teaching of Communication. Vol. II. Writing: Process, Development and Communication, Hillsdale, L. E. A., 198 1 .

Freedie, R. O.: Discours Production and Comprehension. Norwood, Ablex, 1977.

Freedi.e, R. O.: New Directions in Discourse Processing. Norwood, Ablex, 1979.

FREEDMAN, S. W.: "Student characteristics and essay tests writing performance». Research in the Teacbing of English, 1983, 17 (4), 313-329.

Frith, U.: «Reading by eye and writing by ear». En Kol.ers, P. A.; Wrol.STAD, M., y BouMA, H. (eds.): Processing of visible language. Plenum Press, 1980.

GeI.B, I. J.: $A$ study of priting. Chicago, University Chicago Press, 1952.

GiBson, J. E., y Levin, H.: The psychology of reading. Cambridge, The MIT Press, 1978.

Gil. G., y Santana, B.: “Los modelos del proceso de la escritura». Estudios de Psicologia, 1985, 19-20.

Gili.iNGHAM, A., y STIL.Lian, B.: Remedial teaching for children with specific difficulty in reading, spelling and penmanship. Cambridge, Educators Publishing Serie, 1977.

GOES, C. DE, y MARTLEW, M.: «Beginning to read and write; an exploratory study of young children's understanding of metalinguistic terms and graphic conventions». First Language.

GRAVES, D.: Children's writing: research directions and bypotesis based upon an examination of the writing processes of seven-year-old children. Buffalo, State University of New York at Buffalo, 1983.

GrAVES, D.: "The growth and development of first grade writers." Articulo presentado en la Reunión Anual del Consejo Canadiense de Profesores de Inglés, Ottawa, 1979.

GuNDI.ACH, R. A.: "Children as writers: the beginnings of learning to write». En M. NYSTRAND: What writers know: the language, process and structure of written discourse. Nueva York, Academic Press, 1982.

HAss, W.: Writing without letters. Manchester, Manchester University Press, 1976.

HAIIEY, J.: Teacbing writing K-8. Berkeley, Instructional Laboratory, University of California, 1978.

HARROP, A., y MCC AN, C.: «Modifying "creative writing" in the classroom". British Journal Educational Psychology, $1984,14,62-72$.

HALSNER, R. M.: "Interaction of selected personality factors and teachers comments in a sequentially-developed composition curriculum». Fordham University, 1975.

HeNDerson, L.: Ortography and word recognition. Academic Press, 1982. 
KAY'FETZ, J. L.: «Natural sequences in speech and writing in adult second language acquisition». System, $1982,10(2), 145-157$.

KEI.I.Y, J. F., y WHITEHEAD, R. L.: «Integrated spoken and written english instruction for the hearing-impaired student". Journal of Speech and Hearing Disorders, 1983, 48, 41 5-422.

KinNEAVY, J. L.: A Theory of Discourse. Englewood-Cliffs, 1971.

KRAMER, H. W.: The relationship between personality type and achievement in expository and creative writing. The University of Michigan, 1977.

KROL.., B. M., y VANN, J. R. (eds.): Exploring speaking-writing relationships. Urbana, National Council of Teachers of English, 1981 .

LANGER, J. A.: "The effects of available information on responses to school writing tasks". Research in the Teaching of English, 1984, 18 (1), 27-44.

LOBROT, M.: Troubles de la langue écrite et remedes. Paris, Les Editions ESF, 1972.

LUNDSTEEN, S.: Children learn to communicate: language arts througb creative problem solving. Englewood Cliffs, Prentice-Hall, 1976.

MARCIE, P.: "Writing disorders associated with focal cortical lesions». En M MARTLEW: The psycbology of written language. A development approach. Londres, John Wiley and Sons, 1983.

MARTLEW, M.: The psychology of written language. A developmental approach. Londres, John Wiley and Sons, 1983.

MOSEnthal., P.; TAMOR, L., y WALMSIEY, S.: Research on writing: principles and methods. Longman, 1983.

MYKLEBuST, H. R.: The psychology of deafness. Nueva York, Grune and Stratton, 1964.

MYki.eBuST, H. R.: Pictory Story Language Test. Nueva York, Grune and Stratton, 196s.

MYKI.EBUST, H. R.: Development and disorders of written language. Vols. Iy II. Londres, Grune and Stratton, 1973.

NAUCI.ER, K.: «Connections between spoken and written language evidence from three investigations on normal and pathological written performance». Journal of Pragmatics, 1983, 7, 595-602.

NYSTR AND, M.: What writers know: the language, process and structure of written discourse. Nueva York, Academic Press, 1982.

Nystrand, M.: "The structure of Textual space». En M. Nystrand (Ed.) What writers know. The language, process and structure of written discourse. Cap. 3. Nueva York, Academic Press, 1982.

Ol.lvaux, R.: Désordres et réeducation de l'écriture. Paris, Edition ESF, 1971.

OWENS, L.: "Syntax in children's written composition, socioeconomic status and cognitive development». The Australian Journal of Education, 1976, 20 (2), 202-222.

Paivio, A., y BegG, I.: Psychology of language. Englewood Cliffs, Prentice-Hall, 1981 .

PERI, S.: "Five writers writing: case studies of the composing processes of unskilled college writers". Nueva York University, $197^{8}$.

Phel.Ps-GunN, T., y Phei.PS-Terasaki, D.: Written language instruction. Rockville, Aspen System Corporation, 1982 .

POOLE, M.: "Socioeconomic status and written language». En M. MARTI.EW: The psychology of written language. A developmental approach. Londres, John Wiley and Sons, 1983.

POOLE, M. E.: «Social class differences in code elaboration: a study of written communication at the tertiary levelm. Australian and New Zeland Journal of Sociology, 1972, 8, 46-55.

READ, C.: "Writing is not the inverse of reading for young children". En C. H. FREDERIKSEN y J. F. DOMINIC (eds.): Writing: the nature, development and teaching of written communication. Vol. I: Writing: process, development and communication. Hillsdale, Erlbaum, 1981 .

ROSENTHAL, J. H.: The Neuropsychopathology of written language. Chicago, Nelson-Hall, 1977.

RuBiN, A.: "A theoretical taxonomy of the differences between oral and written language». Technical report. Champaign, Center for the Study of Reading, Universidad de Illinois, 1978.

RUSSEI, D. H.: Characteristics of good and poor spellers. Bureau of Publications of the Columbia University, 1937.

SALMINEN, J.:Die spracblicbe Entwiklung des Kindes und die Voraussetzungen zum Erlernen des Lesens und Rechtscbreibens. Joensuum Korkeakoulu, 1979.

SChAilert, D. L.; KI.EIMAN, G. M., y Rubin, A. D.: "Analysis of differences between written and oral languagem. Technical Report, Champaign, Center for the Study of Reading, 1977.

SMiтH, W.: «Reading and writing». Articulo presentado en la Conferencia de la Asociación Internacional de Lectura, Atlantic City, N. J., 1971.

SMITH, F.: «Alphabetic writing. A language compromise». En SMITH, F.: Psycbolinguistics and reading. Nueva York, Holt, Rinehart and Winston, 1973.

SovIK, N.: Developmental cybernetics of bandwriting and graphic behavior: an experimental system analysis of writing readiness and instruction. Oslo, Universitetsforlaget, 1979.

STALlaRD, CH. K.: "An analysis of the writing behavior of good student writers". Research in the Teaching of English, 1974, 8(2), is 6-163.

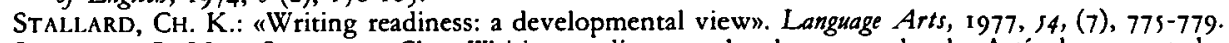

STAl.t.ARD, L. M., y Staliard, Ch.: «Writing readiness at the elementary level». Artículo presentado en la Reunión Anual de la Conferencia de la Escuela Secundaria Inglesa y la Conferencia de la Educación Inglesa», Omaha, marzo $27-29,1980$.

StANFORD, G., y.Smith, M.: "Objetive evaluation on english composition». The Louisiana State University and Agricultural and Mechanical College, 1976 .

STuBBS, M.: Language and literacy: the sociolinguistics of reading and writing. Routledge and Kegan Paul, 1980. 
STUBвS, M.: "Can I have that in writing, please? Some neglected topics in speech act theory". Journal of Pragmatics, $1983,7,479-494$.

TAMOR, L.; BOND, J. T., y MATZ, R. D.: «Instruction writing: a promision approach to the study and teaching of written compositionm. Artículo presentạdo en la Reunión de la Asociación Ameticaña de Investigación Educativa, Boston, abril 1980.

TANnEN, D.: Oral and literate strategies in spoken and written discourse. Michigan, Ann Arbor, 1981.

TANNEN, D. (ed.): Spoken and written discourse. Hillsdale, Ablex, 1982.

Temple, Ch.; NAthan, R., y BURRIS, N.: The beginnings of writing. Allyn y Bacon, 1982.

TrOsKY, O. S., y WOOD, C. C.: "Using a writing model to teach reading». Journal of Reading, 1982, 26 (1), 34. TSVETKOVA, L. S.: Reeducación del lenguaje, la lectura y la escritura. Barcelona, Edit. Fontanella, 1977.

VACHEK, J.: Written language: general problems and problems of english. Mouton, The Hague, 1973.

VAN DijK, T. A.: Text and Context. Explorations in the Semantics and Pragmatics of Discourse. Londres, Longman, 1977.

VYGOTSKY, L. S.: "The prehistory of written language». En M. MARTI.EW: Tbe psychology of written language. A developmental approach. Londres, John Wiley and Sons, 1983.

WEIGL, E.: "On written language. Its acquisition and its alexic-agraphic disturbances». En E. LENNEBERG y LENNEBERG: Foundations of language development a multidisciplinary approach. Nueva York, Academic Press, 1979.

WISEMAN, D.: «Helping children take early steps toward reading and writing». The Reading Teacher, 1984, $37(4), 340-344$.

\section{Resumen}

En este trabajo, tras una breve introducción en la que se considera la naturaleza $e$ importancia de la escritura, se exponen las ideas generales sobre el estudio de su desarrollo, tanto desde un punto de vista bistórico como ontogenético. Seguidamente, se propone una clasificación de los campos de estudio psicológico de la escritura. Por ultimo, se expone un posible marco integrador del área de estudio de la escritura desde un punto de vista cognitivo.

\section{Summary}

In this paper, after a short introduction which considers the nature and importance of writing, the main ideas about the study of it's development, from an historical perspective as well as from an ontogenetical point of view, are exposed. Next, a classification of the different topics of the psycbological study of writing is proposed. Finally, a feasible integrative framework of the areas of study on writing from a cognitive perspective is offered. 